\title{
Influence of Climate on the Proportion of Ixodes ricinus Nymphs and Adults Questing in a Tick Population
}

\author{
JEAN-LUC PERRET, OLIVIER RAIS, AND LISE GERN ${ }^{1}$
}

Institut de Zoologie, University of Neuchâtel, 2007 Neuchâtel 7, Switzerland

\begin{abstract}
J. Med. Entomol. 41(3): 361-365 (2004)
ABSTRACT We studied the relationship between climate and Ixodes ricinus L. tick behavior by following every day the proportion of ticks questing in a tick population placed in polyamide mesh-delimited arenas in the field. Simultaneously, the phenology of the questing density of nymphs and adults was studied by sampling ticks in a close location. At any time during the year, the proportion of questing adults was significantly higher (mean $24 \%$ ) than the proportion of questing nymphs (mean $12 \%)$. The proportion of questing nymphs and adults decreased stepwise with time. The proportion of questing adults partially recovered after each decrease. In contrast, the proportion of questing nymphs was strongly reduced during a single short period in June and did not recover even partially. Decrease in the proportion of questing ticks was strongly related either to a peak in saturation deficit or to a drop in maximal relative humidity. No increase in the proportion of questing nymphs was observed in the arenas during autumn, although an autumn peak of nymphs was observed at the sampling location close to the arenas. This suggests that the autumn peak of nymphs observed in nature was due to newly emerged spring-fed larvae and not to reactivated spring active nymphs.
\end{abstract}

KEY WORDS Ixodes ricinus, questing behavior, climate, phenology

Ixodes ricinus $\mathrm{L}$. is the primary vector of the Lyme borreliosis spirochetes and the tick-borne encephalitis virus in Europe. This tick species has a wide geographical distribution ranging from North Africa to Scandinavia and from Ireland to Russia (Gern and Humair 2002). I. ricinus was found to feed on a large range of vertebrates, including small-, medium-, and largesized mammals, birds, and reptiles (Aeschlimann 1972). Finding a host is a crucial step for the tick life cycle. The host-finding behavior of I. ricinus represents the succession of actions undertaken by unfed ticks to get a successful blood meal. It is known that $I$. ricinus individuals repeatedly occur at the tips of vegetation during periods called questing. During such periods, ticks wait on the vegetation (Lees and Milne 1951) at variable heights for a potential host (Gigon 1985, Mejlon and Jaenson 1997, Randolph and Storey 1999). During questing, ticks experience various climatic conditions, often under their critical equilibrium humidity, so that they lose water (Needham and Teel 1986). At some point, ticks leave their questing site and move to the litter zone (Lees 1946, Milne 1950, Lees and Milne 1951) to rehydrate by active water sorption (Rudolph and Knülle 1979, Gaede and Knülle 1997; Kahl and Alidousti 1997). Using computer-assisted video tracking, we observed that questing behavior is influenced by climatic conditions: questing duration is inversely related to saturation deficit,

\footnotetext{
${ }^{1}$ E-mail: lise.gern@unine.ch.
}

whereas quiescence duration is not (Perret et al. 2003)

In the field, the density of questing ticks can be estimated by dragging a white flannel flag over low vegetation. It is important to bear in mind that flagging does not sample among ticks in quiescence. Flagging is therefore not a means to estimate tick population density, but only a means to estimate the density of ticks that are questing. Variations in the phenology of I. ricinus (evolution of the questing tick density over time; Jouda et al. 2004) may be associated with major variations in biotic (host species, host density and behavior, and vegetation structure) as well as abiotic factors (climate). Throughout its wide distribution, $I$. ricinus phenology varies considerably (Gray 1991). Indeed, in Algeria, I. ricinus shows a unimodal pattern with maximum nymph density in winter (YousfiMonod and Aeschlimann 1986). In Ireland, I. ricinus nymphs and adults show a bimodal pattern, reaching maximum density in spring and a smaller peak of density in autumn (Gray 1984), whereas in the Crimea, the bimodal phenology reaches its maximum in autumn (Korenberg 2000). In England, both, a unimodal peak with a maximum tick density in spring and bimodal peaks were shown to occur the same year in different sites (Randolph et al. 2002). Finally the phenology of I. ricinus was reported to be variable among years in Sweden (Tälleklint and Jaenson 1996), England (Randolph et al. 2002), and Switzerland (Mer- 
mod et al. 1973, Perret et al. 2000, Perret 2003, Jouda et al. 2004).

The purpose of this work was to study the relationship between climate and tick behavior.

\section{Materials and Methods}

Field Arenas. Six field arenas were built to observe tick activity in the field. These arenas were made of a cylindric polyamide mesh (500- $\mu \mathrm{m}$ grid, Sefar-Nitex, Sefar AG, Haiden, Switzerland) closed with a plastic sheet on the top. The arenas $(30 \mathrm{~cm}$ in height by 2.5 $\mathrm{cm}$ in diameter) were driven into the ground by adding an open 10-cm-long plastic cylinder at the bottom of the arenas. The ground was covered with leaf litter, and introduced ticks had free access to it.

The six arenas were set up in the forest located north of the experimental garden at the University of Neuchâtel botanic garden (coordinates CH1903: $561550 / 205575$, altitude $600 \mathrm{~m}$ ). The forest (deciduous dominant) is situated on the slope of a mountain (Chaumont) north of Neuchâtel with a southern exposure (Jouda et al. 2004). Ticks that were introduced to arenas had been collected in the forest close to the arenas within $48 \mathrm{~h}$ prior and kept at ambient temperature under natural light conditions in a water-saturated atmosphere before the experiment.

In total, 506 nymphs, 54 females, and 83 males were placed in the six arenas (instars were mixed in all arenas). Ticks were placed in three arenas at the end of March 2000 ( 21 March). From the three arenas set up in March, only one could be used for nymphal observation because little holes allowed nymphs (but not adults) to escape. Therefore, 126 nymphs (in arena 1 ) and 77 adults (24 females and 53 males, in arenas 1-3) were observed from 22 March 2000 to 22 December 2000 in arenas 1-3. On 17 May 2000, 180 nymphs and 60 adults ( 30 females and 30 males) were introduced into three additional arenas (4-6) and were observed from 17 May 2000 to 23 October 2000. At that time, 80 newly collected nymphs (collected on the same day in the forest close to the botanical garden) were added to arenas 4 and 5 . Therefore, 260 nymphs and 60 adults were observed in arenas 4 and 5 from 23 October 2000 to 22 December 2000.

The questing behavior of I. ricinus in arenas was observed by counting ticks visible on the sides and top of the arenas. The observer was standing $\approx 40 \mathrm{~cm}$ away from the arenas and stopped breathing while counting ticks. It was assumed that ticks visible above the litter zone were questing. Because ticks had free access to the litter, it was not possible to count the ticks at the bottom of the arenas without disturbing them. The number of ticks visible in the arenas was recorded 216 times (twice daily, in the morning between 8:00 a.m. and 10:00 a.m. and in the afternoon between 1:00 p.m. and 5:00 p.m. over $74 \mathrm{~d}$ and once daily, in the morning, over $68 \mathrm{~d}$ ). The proportion of ticks questing in the arenas over time was calculated by dividing the number of ticks observed at any time by the total number of ticks introduced in the arenas.
Tick Sampling in the Field. To study the tick phenology under natural conditions, we sampled questing $I$. ricinus nymphs and adults by pulling a $1-\mathrm{m}^{2}$ flag over the vegetation every month from January to December 2000. Ticks were collected over a distance of $245 \mathrm{~m}$ each month. The flag was examined for ticks every $18 \mathrm{~m}$ The study area is located at an altitude of $585 \mathrm{~m}$ in the same forest where arenas were set up, on the slope of the Chaumont mountain with a southern exposure (coordinates CH1903: 562764/206105) and in the same habitat. Tick sampling took place between 10:00 a.m. and 5:00 p.m.

Climatic Data. Climatic data (temperature and air humidity for each hour) were provided by the Swiss Meteorological Institute. Data were extracted from the Schweizerische Meteorologische Anstalt 3 database on the "wawona" server at the Swiss Federal Institute of Technology in Zürich, Switzerland, by using a homemade Fortran program. Ten-day average of daily average and maximum relative humidities and $10-\mathrm{d}$ average of daily minimal, average, and maximal saturation deficits were calculated. Saturation deficit, which is a measurement of the "drying power" of the air, was calculated using the empirical formula published elsewhere (Randolph and Storey 1999, and Perret et al. 2000).

Statistical Analysis. Time plots of the proportion of visible ticks (nymphs and adults separately) were created. To highlight the evolution of this proportion, a nonparametric smoothing of the data (Friedman's supersmoother) was applied using the default options of the function "supsmu" of the package "modreg" in R for Linux V0.90.0 (Ikaha and Gentleman 1996). The numbers of visible nymphs and adults were compared using the unpaired Wilcoxon test for each observation of the arenas.

\section{Results}

At any observation time during the study period, the proportion of questing adults was significantly higher (mean 24\%) than the proportion of questing nymphs (mean 12\%) $(P<0.000001$, Wilcoxon tests $)$. The proportion of questing ticks (nymphs and adults) decreased stepwise with time (Fig. 1). The proportion of visible adults partially recovered after each decrease (Fig. 1a). In contrast, the proportion of visible nymphs decreased substantially during a single short period in June and did not recover even partially (Fig. 1b).

Decrease in the proportion of visible ticks was strongly related either to a peak in saturation deficit or to a drop in maximal relative humidity (Fig. 1, dotted arrows). In autumn, no increase in the proportion of questing ticks occurred as saturation deficit decreased. However, a proportion of the 80 nymphs collected and introduced to the arenas in October were observed questing in the arenas (Fig. 1).

Our observation of I. ricinus ticks in the field showed a significant increase in questing tick density in March, for both nymphs and adults, and the peak of questing tick density was reached at the end of April. This peak was followed by a decrease in density, reaching its lowest level in July. A second peak of questing density for 


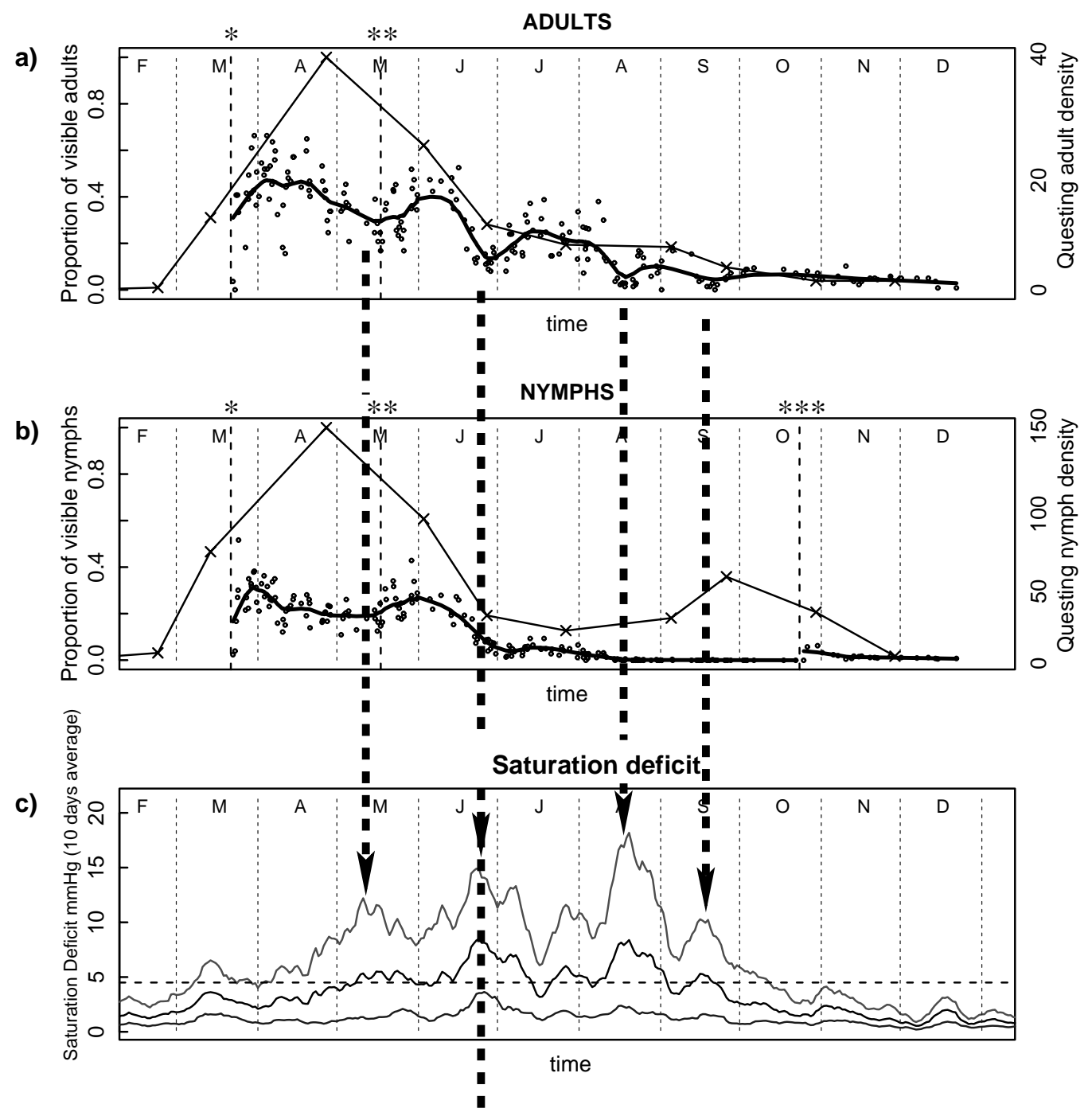

d)

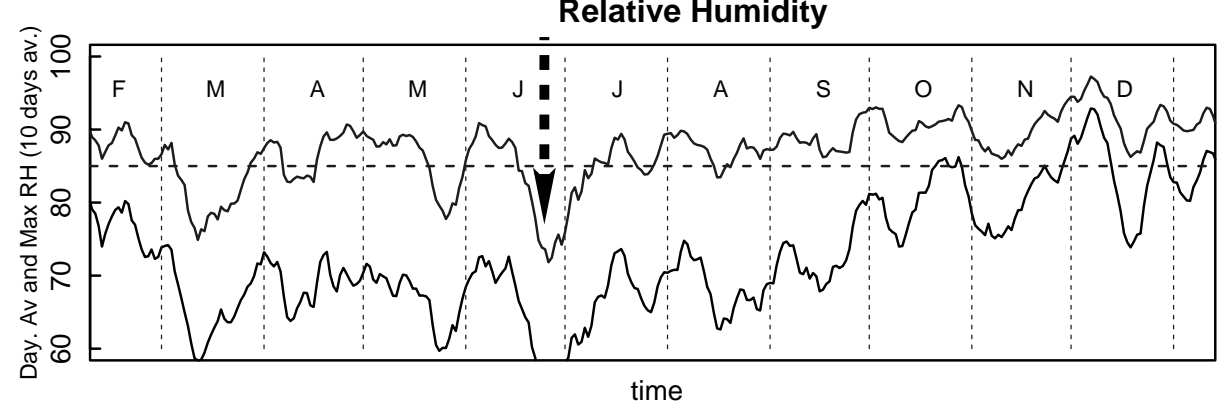

Fig. 1. Proportion of visible I. ricinus adults and nymphs in field arenas, questing nymphs and adults density (number of ticks per $100 \mathrm{~m}^{2}$ sampled in nature), and average climatic conditions recorded in 2000 , including density of questing adults (thin line) and proportion of visible adults (bold line, the continuous line is a nonparametric smoothing of the data) (a); density of questing nymphs (thin line) and proportion of visible nymphs (bold line, the continuous line is a nonparametric smoothing of the data (b); 10-d average of daily minimal, average, and maximal saturation deficit (dashed line, $4.5 \mathrm{mmHg}$ saturation deficit) (c); and 10-d average of daily average and maximum relative humidity (dashed line, $85 \%$ RH) (d). Introductions of ticks in the arenas are indicated by dotted vertical lines. March, April, and early May nymphal data are from one arena (1) due to escape of nymphs from arenas 2 and 3. *, first introduction of ticks to arenas. **, second introduction of ticks to arenas. $* * *$, third introduction of ticks to arenas. 
nymphs was observed at the end of September (Fig. 1b), whereas adult density continued to decrease (Fig. la).

\section{Discussion}

Results obtained in this study confirm that the questing behavior of I. ricinus, as measured by the proportion of ticks questing at any given sampling period, is influenced by climate (Perret et al., 2000, 2003; Randolph et al., 2002) and show that climate influences the proportion of ticks questing.

The proportion of questing ticks decreased irregularly from April to September. Ticks in the arenas were protected from predators and could not be picked up by hosts. Therefore, the general decrease in tick number was probably due to tick death by exhaustion of their energy reserves (Steele and Randolph 1985, Randolph and Storey 1999), the tick life span being limited by energy that they obtain only from blood meals (one per developmental stage). Because ticks in the arenas could not select microhabitats more suitable to quest or to rehydrate, this might have increased tick mortality. The ground coverage by the litter did not allow counts of all living ticks. Such counts would have disturbed tick behavior, and great care was taken to minimize the stimulation induced by the regular counts of questing ticks. To achieve the time resolution allowing for observation the relation of tick activity with weather conditions, we rather related the number of questing ticks to the initial number of ticks introduced in the arenas, and we were interested in the way the proportion of questing ticks changed in relation with climate.

The decrease in the proportion of questing ticks was influenced by drought. Indeed, the proportion of questing ticks dropped considerably after the first large drought in June. Thus, abrupt declines in questing ticks coincided with variation in climatic conditions. This finding had been previously hypothesized from flag sampling studies in the same forest (Perret et al. 2000) and in the United Kingdom (Randolph et al. 2002). Drought events consisted either in drops in the maximum relative humidity (mostly occurring at night) or in increases of the maximum saturation deficit (mostly occurring during the day). Although the top of the arenas was covered with a plastic sheet, we do not expect this aspect of the arena's construction to affect the microclimatic conditions within the arenas, except maybe for the direct impact of water falling from the trees. Indeed, the diameter of the plastic sheet was only $2.5 \mathrm{~cm}$ and was regularly found to be covered with water on both sides after rain. Because the arenas were protected from the direct impact of the sun from the trees above, we do not expect any greenhouse effect from the plastic sheet. The observed impact of climate on tick questing can be explained by two factors: the reduced maximal relative humidity prevented ticks from rehydrating at their questing site during the night, or the increased saturation deficit during the warmer periods of the day increased water loss at questing sites, thus forcing ticks to search for a humid place to rehydrate.
In the days after the introduction of the second group of ticks, the proportion of questing ticks increased slightly, probably only among the newly introduced ticks. Before their introduction in the arenas, these ticks were held in humid atmosphere and were therefore in optimal condition for questing.

Adult activity was prolonged until September, whereas nymphal activity strongly decreased in June. Interestingly, the proportion of questing adults, but not nymphs, was partially restored after each drought. These differences may be explained by the bigger size of adults, which are less susceptible to desiccation (MacLeod 1935, Lees 1946) and which possess higher absolute energy reserves than nymphs (Randolph et al. 2002; personal communication). In addition, nymphs and adults did not immediately react to climatic conditions. In fact, no significant variation in the proportion of questing ticks was observed between the morning and the end of the afternoon. This finding shows that in nature, ticks use their water content as a buffer to survive desiccating conditions for short periods. Adults, due to their size, have a higher water content than nymphs. In addition, the surface-to-volume ratio is smaller for adults than nymphs, so that adults lose proportionally less water than nymphs. More energy, higher water content, and smaller water loss in adult ticks enabled a higher proportion of adults to quest for longer periods than nymphs. Buffered reactions of ticks to climate were also reported by Gigon (1985), who observed that questing was influenced by a combination of relative humidity and temperature.

In 2000, the density of questing nymphal and adult ticks in the field declined at the end of May, during the first drought event. A similar decline was observed in 2000 in another location close to our study site (Chaumont Low) (Jouda et al. 2004). In both natural sites, the questing tick density decreased before the strong decrease (June) in the proportion of questing ticks in the arenas. This suggests that either the decrease in the density of questing ticks in the field was due to a reduction in the tick population size (output of ticks) rather than a decrease in the proportion of questing ticks or that different cohorts of ticks coexist in the field, following different questing histories, so that the behavior of the single group of ticks studied in the arenas was different from that in the mixed population. However, because ticks observed in the arenas were collected very early in the season, it is not expected that these ticks would quest longer than ticks present in the field later in spring. Therefore, we think that the decline in questing ticks in the field in the two locations close to the arenas during May 2000 in Neuchâtel (present data, Jouda et al. 2004) was primarily due to tick removal, either by hosts or by predation of ticks (Steele and Randolph 1985, Ginsberg and Zhioua 1999) or by dispersal of ticks (Carroll and Schmidtmann 1996, Perret et al., 2003) rather than by limitation of the proportion of questing ticks by climatic conditions.

No increase in the proportion of questing nymphs was observed in the arenas during autumn 2000 . However, an autumn peak of nymphs was observed at the 
two sampling locations close to the arenas (present data, Jouda et al. 2004). This suggests that the autumn peak of nymphs observed in nature during 2000 was due to newly emerged spring-fed larvae and not to reactivated spring active nymphs. The presence of an autumn peak of nymphs in a close location in 2000 was related to warm summer temperature (Jouda et al. 2004). In fact, the year 2000 was a warm year in Neuchâtel compared with 1999 and 2001 (Jouda et al. 2004). The warmer temperature may have allowed spring-summer-fed larvae to molt and emerge the same year, as reported by Chmela (1969). Randolph et al. (2002) hypothesized that the occurrence of an autumn peak in tick activity is dependent upon the development duration of spring fed ticks. Our results in the arenas strengthen this explanation.

\section{Acknowledgments}

We thank François Felber for kindly allowing us to work in the Botanical garden in Neuchâtel. This article is part of the Ph.D. dissertation of J.-L.P. This work was financially supported by the Swiss National Science Foundation (3257098.99).

\section{References Cited}

Aeschlimann, A. 1972. Ixodes ricinus, Linné, 1758 (Ixodoidea; Ixodidae) essai préliminaire de synthèse sur la biologie de cette espèce en Suisse. Acta Trop. 29: 321-340.

Carroll, J. F., and E. T. Schmidtmann. 1996. Dispersal of blacklegged tick (Acari: Ixodidae) nymphs and adults at the woods-pasture interface. J. Med. Entomol. 33: 554558.

Chmela, J. 1969. On the developmental cycle of the common tick (Ixodes ricinus L.) in the North-Moravian natural focus of tick-borne encephalitis. Folia Parasitol. 16: 313-319.

Gaede, K., and W. Knülle. 1997. On the mechanism of water vapour sorption from unsaturated atmospheres by ticks. J. Exp. Biol. 200: 1491-1498.

Gern, L. and P.-F. Humair. 2002. Ecology of Borrelia burgdorferi sensu lato in Europe, pp. 149-174. In Lyme borreliosis: biology, epidemiology and control. CAB, Wallingford, United Kingdom.

Gigon, F. 1985. Biologie d'Ixodes ricinus sur le Plateau suisse. Ph.D. dissertation, Université de Neuchâtel, Switzerland.

Ginsberg, H. S., and E. Zhioua. 1999. Influence of deer abundance on the abundance of questing adult Ixodes scapularis (Acari: Ixodidae). J. Med. Entomol. 36: $376-$ 381.

Gray, J. S. 1984. Studies on the dynamics of active populations of the sheep tick, Ixodes ricinus L. in co. Wicklow, Ireland. Acarologia 25: 167-178.

Gray, J. S. 1991. The development and seasonal activity of the tick Ixodes ricinus: a vector of Lyme borreliosis. Rev. Med. Vet. Entomol. 79: 323-333.

Ikaha, R., and R. Gentleman. 1996. A language for data analysis and graphics. J. Comput. Graph. Stat. 5: 299-314.

Jouda, F., J.-L. Perret, and L. Gern. 2004. Influence of altitude on Lxodes ricinus density, and on distribution and prevalence of Borrelia burgdorferi sensu lato infection. J. Med. Entomol. 41: 162-169.

Kahl, O., and I. Alidousti. 1997. Bodies of liquid water as a source of water gain for Ixodes ricinus (Acari: Ixodidae). Exp. Appl. Acarol. 21: 731-746.
Korenberg, E. I. 2000. Seasonal population dynamics of $I x$ odes ticks and tick-borne encephalitis virus. Exp. Appl. Acarol. 24: 665-681.

Lees, A. D. 1946. The water balance in Ixodes ricinus L. and certain other species of ticks. Parasitology 37: 1-20.

Lees, A. D., and A. Milne. 1951. The seasonal and diurnal activities of individual sheep ticks (Ixodes ricinus L.). Parasitology 41: 189-208.

MacLeod, J. 1935. Ixodes ricinus in relation to its physical environment. II. The factors governing survival and activity. Parasitology 27: 123-244.

Mejlon, H. A., and T. Jaenson. 1997. Questing behaviour of Ixodes ricinus ticks (Acari: Ixodidae). Exp. Appl. Acarol. 21: $747-754$.

Mermod, C., A. Aeschlimann, and J.-F. Graf. 1973. Ecologie et éthologie d'Ixodes ricinus Linné 1758, en Suisse (Acarina, Ixodoidea): première note: fluctuations numériques. Acarologia 15: 197-205.

Milne, A. 1950. The ecology of the sheep tick, Ixodes ricinus L. Microhabitat economy of the adult tick. Parasitology 40: $14-34$.

Needham, G. and P. Teel. 1986. Water balance by ticks between bloodmeals, pp. 100-151. In J. R. Sauer and J. A. Hair [eds.], Morphology, physiology and behavioral biology of ticks, Ellis Horwood Series in Acarology, Chichester. E. Horwood, Chichester, West Sussex, England.

Perret, J.-L. 2003. Computer-assisted laboratory observations and field studies of the host-finding behaviours of the tick Ixodes ricinus (Acarina: Ixodidae): ecological implications of climate and light. Ph.D. dissertation, University of Neuchâtel, Switzerland.

Perret, J.-L., E. Guigoz, O. Rais, and L. Gern. 2000. Influence of saturation deficit and temperature on Ixodes ricinus tick questing activity in a Lyme borreliosis-endemic area (Switzerland). Parasitol. Res. 86: 554-557.

Perret J.-L., P. Guerin, P.-A. Diehl, M. Vlimant, and L. Gern. 2003. Darkness favours mobility and saturation deficit limits questing duration in Ixodes ricinus, the tick vector of Lyme disease in Europe. J. Exp. Biol. 206: 1809-1815.

Randolph, S. E., and K. Storey. 1999. Impact of microclimate on immature tick-rodent host interactions (Acari: Ixodidae): implications for parasite transmission. J. Med. Entomol. 36: 741-748.

Randolph, S. E., R. M. Green, A. N. Hoodless, and M. F. Peacey. 2002. An empirical quantitative framework for the seasonal population dynamics of the tick Ixodes ricinus. Int. J. Parasitol. 32: 979-989.

Rudolph, D., and W. Knülle. 1979. Mechanisms contributing to water balance in non-feeding ticks and their ecological implications. Rec. Adv. Acarol. 1: 375-383.

Steele, G. M., and S. E. Randolph. 1985. An experimental evaluation of conventional control measures against the sheep tick Ixodes ricinus L. (Acari: Ixodidae). I. A unimodal seasonal activity pattern. Bull. Entomol. Res. 75: $489-499$.

Tälleklint, L., and T. G. Jaenson. 1996. Seasonal variations in density of questing Ixodes ricinus (Acari: Ixodidae) nymphs and prevalence of infection with Borrelia burgdorferi s. l. in south central Sweden. J. Med. Entomol. 33: 592.

Yousfi-Monod, R., and A. Aeschlimann. 1986. Recherches sur les tiques (Acarina: Ixodidae), parasites de bovidés dans l'ouest algérien. 1. Inventaire systématique et dynamique saisonnière. Ann. Parasitol. Humaine Comparée 61: 341-358.

Received 13 August 2003; accepted 24 December 2003. 